\title{
Article \\ Synthesis and Hydrophilicity Analysis of bis(propane-1,2-diol) Terminated Polydimethylsiloxanes (PDMSs)
}

\author{
Lan-Hee Yang, Kyeong Eun Park and Sungho Yoon* \\ Department of Chemistry, Chung-Ang University, 84 Heukseok-ro, Dongjak-gu, Seoul 06974, Korea; \\ yangd5d5@cau.ac.kr (L.-H.Y.); pke8597@cau.ac.kr (K.E.P.) \\ * Correspondence: sunghoyoon@cau.ac.kr
}

Citation: Yang, L.-H.; Park, K.E.; Yoon, S. Synthesis and Hydrophilicity Analysis of bis(propane-1,2-diol) Terminated Polydimethylsiloxanes (PDMSs). Materials 2022, 15, 753. https://doi.org/10.3390/ ma15030753

Academic Editors: Cecilia Mortalò, Valentina Zin and Silvia Deambrosis

Received: 29 November 2021

Accepted: 14 January 2022

Published: 19 January 2022

Publisher's Note: MDPI stays neutral with regard to jurisdictional claims in published maps and institutional affiliations.

Copyright: (C) 2022 by the authors. Licensee MDPI, Basel, Switzerland. This article is an open access article distributed under the terms and conditions of the Creative Commons Attribution (CC BY) license (https:// creativecommons.org/licenses/by/ $4.0 /)$.

\begin{abstract}
Among silicone oligomers, polydimethylsiloxane (PDMS) is widely used industrially and has the advantage of improving the properties of other compounds, such as flame-retardant polyurethane (PU). However, as there are barriers to the synthesis of PU-grafted siloxane, owing to the polarity difference between isocyanate and PDMS, numerous research efforts are being aimed at improving the hydrophilicity of PDMS. To improve the hydrophilicity and reactivity of hydroxyl PDMS, bis(propane-1,2-diol)-terminated PDMS (G-PDMS-G) with four hydroxy (-OH) groups was synthesized through ring-opening addition to replace both ends of linear $\alpha, \omega$-hydroxyl PDMS (HO-PDMS-OH) with glycidol, resulting in hydrophilic PDMS rather than dihydroxy PDMS. In all cases of G-PDMS-G, the contact angle and viscosity both decreased by more than $20 \%$, confirming the improved hydrophilicity. In particular, G-PDMS-G-3, which has the largest molecular weight, demonstrated the greatest decrease in viscosity and contact angle (33\%).
\end{abstract}

Keywords: polydimethylsiloxane (PDMS); hydrophilicity; molecular weight; viscosity; contact angle

\section{Introduction}

Polydimethylsiloxane (PDMS) is the most common and widely used silicone compound and is considered an important material, with diverse advantages such as high thermal stability, low glass transition temperature, low surface energy, high molecular flexibility, and biocompatibility. This polymer has been widely used in industry as a lubricant, antifoaming agent, and resin for soft lithography. In addition, it can be applied to improve the properties of other compounds [1-3].

One application of PDMS is in flame-retardant polyurethane (PU). This siliconeincorporated PU is non-corrosive, and it is less toxic and generates less smoke when exposed to fire than phosphorus and halogen-based flame retardants [4-6]. In addition, it can also be used as a coating agent due to its low surface energy [7,8]. However, PU is a block copolymer with urethane repeats, synthesized by the reaction between isocyanate and polyol molecules, and should contain two or more isocyanate groups and hydroxyl groups for high reactivity. The polar urethane hard segment and non-polar siloxane soft segment in the PU drive phase separation.

Diverse studies on the synthesis of silicone polymers with improved hydrophilicity have been conducted over the last two decades for various purposes [9-12]. In general, hydrophilic polysiloxanes for perfumes, shampoos, and paint additives are synthesized by a hydrosilylation reaction according to the graft method with a linear copolymer or polyether chain on the PDMS backbone [13-15]. In addition, there are reports on the development of biopolymers with hydrophilic groups that are easily biodegradable and non-toxic to humans $[16,17]$. Other studies demonstrate that increasing the number of hydrophilic saccharides bound to the PDMS backbone or combining oligosaccharides rather than monosaccharides increases hydrophilicity $[18,19]$. However, when using the graft method, it is difficult to control the branches located along the PDMS backbone. Therefore, 
Zhang et al. synthesized PDMS with a diol group at one chain end by hydrosilylation of 1,2-allyl glycidol ether using a termination method [20,21].

In this study, bis(propane-1,2-diol)-terminated PDMS (G-PDMS-G), which has a symmetrical structure and includes diol groups at both ends (Figure 1B), was prepared by the terminal method to achieve a higher hydrophilicity and reactivity than those for HO-PDMS$\mathrm{OH}$ (Figure 1A). Additionally, PDMS containing four OH groups was synthesized by a facile post-treatment process involving hydrosilylation, without catalyst, and analyzed for improved hydrophilicity.

(A) $2 \mathrm{R}-\mathrm{OCN}+\mathrm{HO}-\mathrm{PDMS}-\mathrm{OH} \rightarrow$<smiles>[R]NC(=O)OCC(C)CCSC(=O)N[R]</smiles><smiles>C1CCCC1</smiles>

(B)
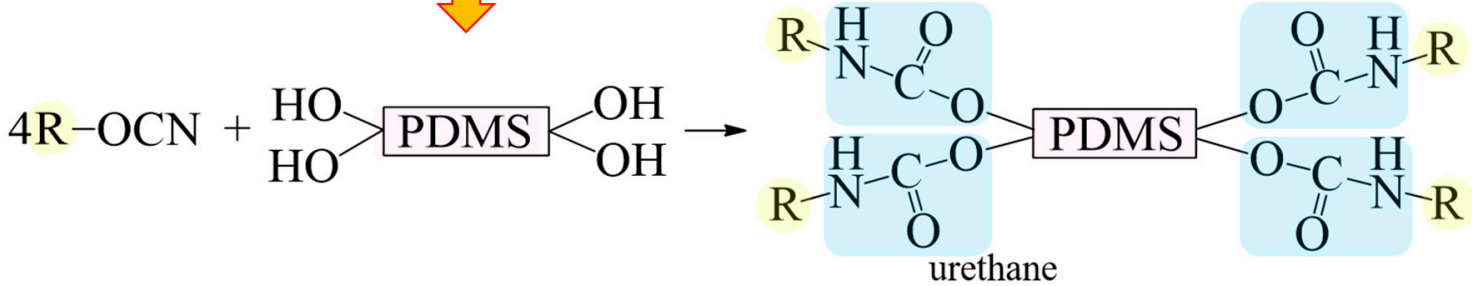

\section{Isocyanate Siloxane polyol}

\section{Polyurethane}

Figure 1. Schematic diagram of reaction between isocyanate and siloxane polyol for synthesis of PU. (A) Synthesis of PU using siloxane polyol with two hydroxyl groups, producing two urethane groups. (B) Synthetic reaction from a siloxane polyol with four hydroxyl groups, resulting in four urethane groups.

\section{Experimental}

\subsection{Materials}

PDMS hydroxyl-terminated (number average molecular weight (Mn): 550, viscosity: $\sim 25 \mathrm{cSt}$ ), PDMS hydroxyl-terminated (viscosity: $\sim 65 \mathrm{cSt}$ ), sodium hydride (dry, 90\%), glycidol (96\%), and tetrahydrofuran (anhydrous, $>99.9 \%$, inhibitor-free) were purchased from Merck Korea (Seoul, Korea). PDMS hydroxyl-terminated (weight-average molecular weight (Mw): 4200) was obtained from Alfa Aesar (Ward Hill, MA, USA).

\subsection{Sample Characterization}

${ }^{1} \mathrm{H}$ NMR spectra of the products were measured with a Varian $600 \mathrm{MHz}$ NMR spectrometer (Palo Alto, CA, USA) using $\mathrm{CDCl}_{3}$ as the solvent. The molecular weight and polydispersity index (PDI) were determined by gel permeation chromatography (GPC) using a Waters 2410 refractive index detector (Milford, MA, USA) with a Waters 515 HPLC pump. The column was eluted with THF at a flow rate of $1.00 \mathrm{~mL} / \mathrm{min}$. GPC curves were calibrated using a polystyrene standard with molecular weights ranging from 162 to $6,570,000 \mathrm{~g} \mathrm{~mol}^{-1}$. Dihydroxy-terminated PDMS sample was coated on a $1.5 \mathrm{~cm} \times$ $1.5 \mathrm{~cm}$ glass slide washed with methanol. The contact angle between the $4 \mu \mathrm{L}$ DI water droplets and the surface of the modified samples was measured using an SEO Phoenix-300 Touch (Kromtek, Malaysia). The viscosity of the neat samples was measured directly by a CPA-41Z spindle using a Brookfield viscometer (DV2TLVCJ0) (Boston, MA, USA).

\subsection{Glycidol-Terminated PDMS (G-PDMS-G-1) from HO-PDMS-OH-1}

A PDMS hydroxyl-terminated solution (10 g, $18.18 \mathrm{mmol}, \mathrm{Mn}: \sim 550$, viscosity: $25 \mathrm{cSt}$ ) in THF $(100 \mathrm{~mL})$ was cooled in an ice bath under an $\mathrm{N} 2$ atmosphere. Then, sodium hydride (36.36 mmol) was slowly added to the solution. The solution, which turned beige with $\mathrm{H} 2$ generation, was stirred until clear. Glycidol $(28.17 \mathrm{mmol})$ was injected and reacted for $3 \mathrm{~h}$ 
at room temperature. Distilled water (DW) was used to quench the reaction. The product solution was washed three times with excess DW and the water was removed (yield: $60 \%$ ).

\subsection{Glycidol-Terminated PDMS (G-PDMS-G-2) from HO-PDMS-OH-2}

PDMS hydroxyl-terminated solution (10 g, $3.030 \mathrm{mmol}$, viscosity: 65 cSt) in THF $(100 \mathrm{~mL})$ was cooled in an ice bath under an N2 atmosphere. Sodium hydride $(6.060 \mathrm{mmol})$ was injected into the solution and stirred until the solution color changed from beige to clear. Glycidol (12.12 mmol) was added and reacted at room temperature. After $3 \mathrm{~h}, \mathrm{DW}$ was injected to quench the reaction. Finally, the product solution was washed three times with excess DW and separated (yield: 63\%).

\subsection{Glycidol-Terminated PDMS (G-PDMS-G-3) from HO-PDMS-OH-3}

PDMS hydroxyl-terminated solution (10 g, $2.381 \mathrm{mmol}, \mathrm{Mw}: ~ 4200)$ in THF (100 mL) was cooled in an ice bath under an $\mathrm{N}_{2}$ atmosphere. Sodium hydride $(4.762 \mathrm{mmol})$ was added to the solution, which turned beige with $\mathrm{H}_{2}$ generation, and the solution was stirred until clear. Glycidol (9.524 mmol) was added and reacted for $3 \mathrm{~h}$ at room temperature. DW was added to quench the reaction. The product solution was washed three times with excess DW and separated (yield: 65\%).

\section{Results and Discussion}

Three types of linear $\alpha, \omega$-dihydroxy PDMS, including HO-PDMS-OH-1 (Mn: 550, viscosity: 25 cSt, Sigma Aldrich, St. Louis, MO, USA), HO-PDMS-OH-2 (viscosity: 65 cSt, Sigma Aldrich), and HO-PDMS-OH-3 (Mw: 4200, Alfa Aesar), were purchased and used as starting materials. As shown in Figure 2, after the dehydration of both ends of the hydroxy PDMS by NaH, the PDMS anion attacks the secondary carbon of glycidol, and ring opening occurs, producing G-PDMS-G-1, G-PDMS-G-2, and G-PDMS-G-3 [22-24].
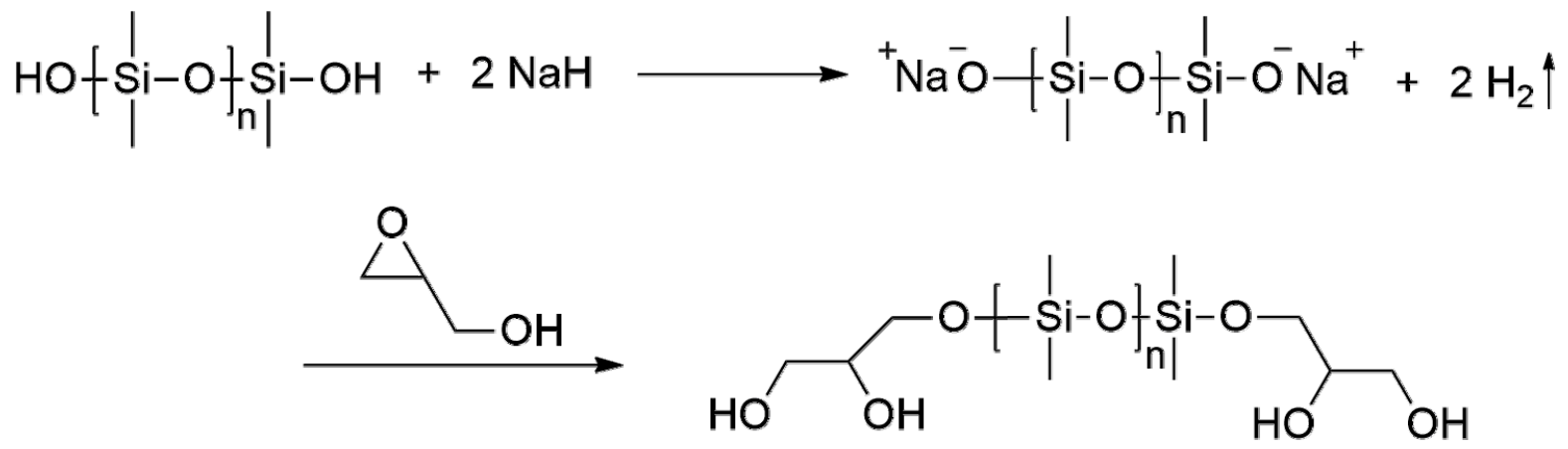

Figure 2. Reaction of dihydroxyl-terminated PDMS (HO-PDMS-OH) to glycidol-terminated PDMS (G-PDMS-G) with sodium hydride.

Figure 3 shows the ${ }^{1} \mathrm{H}$ NMR spectra for the structural comparison analysis of the synthesized PDMS. There is no peak in the ${ }^{1} \mathrm{H}$ NMR spectrum of HO-PDMS-OH in the 2.5-4.5 ppm range (Figure $3 \mathrm{~A}$ ), unlike in the ${ }^{1} \mathrm{H}$ NMR spectrum of glycidol (Figure $3 \mathrm{~B}$ ). However, five chemical shifts were observed in the range of $2.5-4.5 \mathrm{ppm}$ of the ${ }^{1} \mathrm{H}$ NMR spectra of G-PDMS-G-1, G-PDMS-G-2, and G-PDMS-G-3, in which both ends were substituted with glycidol (Figure 3C-E). As shown in Figure 3C, two pairs of geminal protons $\left(\mathrm{H}_{\mathrm{f}} / \mathrm{H}_{\mathrm{g}}\right.$, $\mathrm{H}_{\mathrm{i}} / \mathrm{H}_{\mathrm{j}}$ ) on the secondary carbon out of the five protons, excluding the hydroxyl group, were produced as diastereotopic protons because of the tertiary carbon substituted on the adjacent carbon, which is the chiral center $[25,26]$. As in the case of glycidol, both pairs of geminal protons $\left(\mathrm{H}_{\mathrm{a}} / \mathrm{H}_{\mathrm{b}}, \mathrm{H}_{\mathrm{d}} / \mathrm{H}_{\mathrm{e}}\right)$ are diastereotopic protons (Figure $3 \mathrm{~B}$ ). The chemical shift observed in the 2.5-4.5 ppm range, was not the same as that representing the end of the substituted PDMS. It is likely that glycidol is asymmetrical, with two terminal $\mathrm{OH}$ groups, such as the final G-PDMS-G structure shown in Figure 2. However, it could also be symmetrical and a structure having both. While it is difficult to predict the exact structure 
of the modified glycidol, the C NMR results in Figure S1, from Supplementary Material, point toward the asymmetrical version with the terminal $\mathrm{OH}$ groups.

(A)

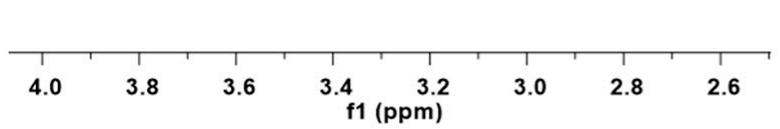

(B)

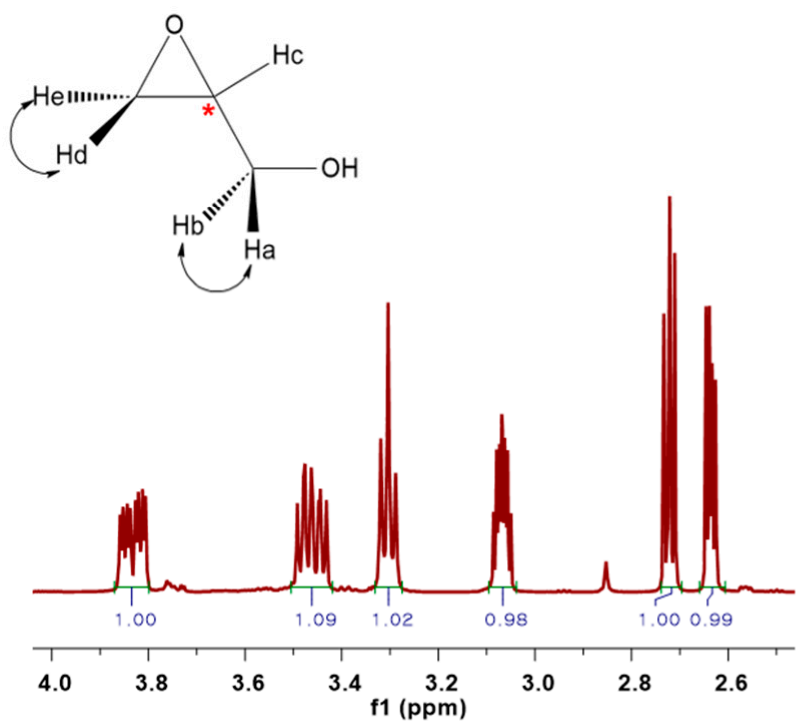

(C)
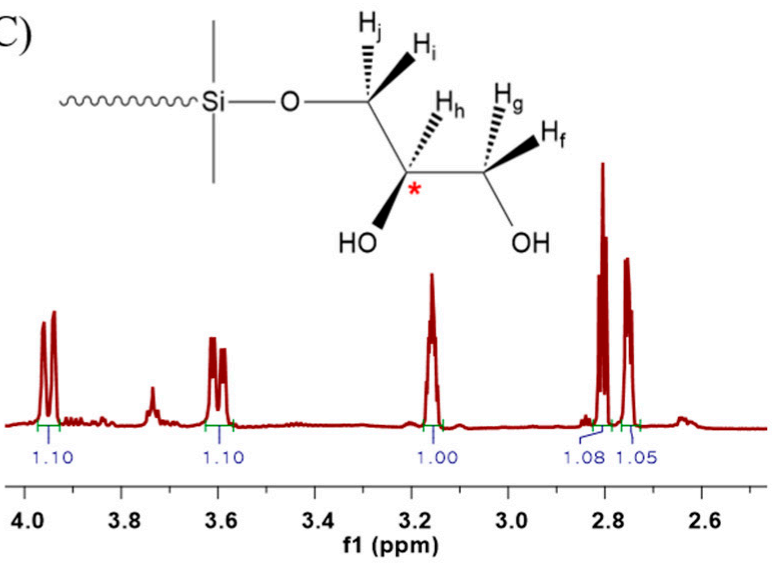

(D)

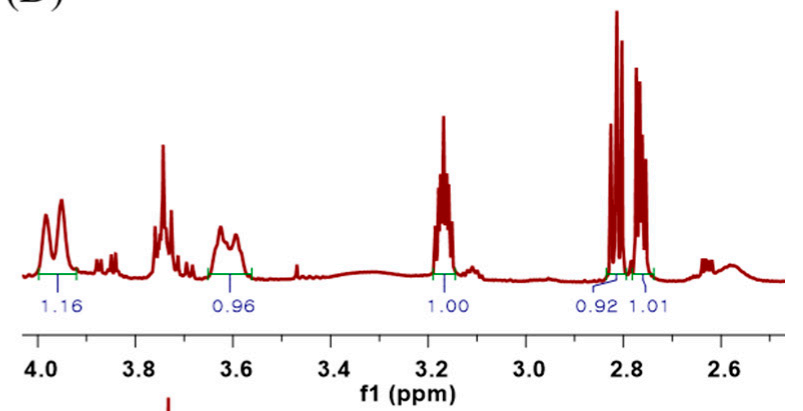

(E)

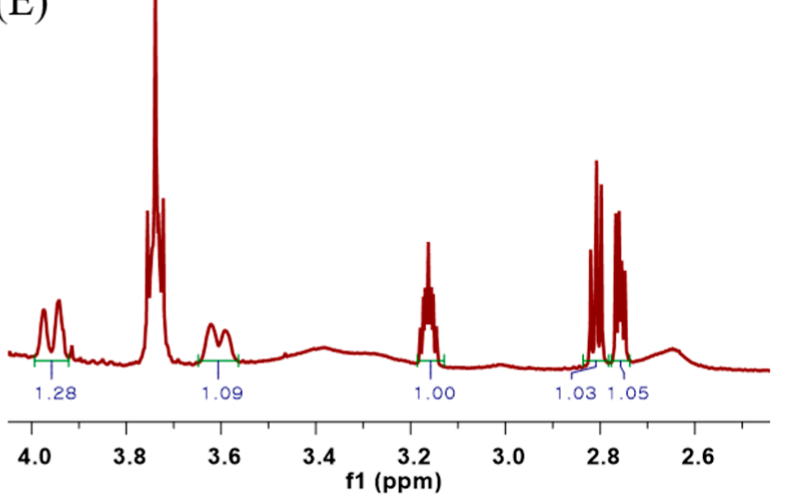

Figure 3. ${ }^{1} \mathrm{H}$ NMR spectra of (A) HO-PDMS-OH, (B) glycidol, (C) G-PDMS-G-1, (D) G-PDMS-G-2, and (E) G-PDMS-G-3.

The experimental ${ }^{1} \mathrm{H}$ NMR and GPC results for the dihydroxy-terminated PDMS (HO-PDMS-OH-1, 2, and 3) starting materials and the PDMS glycidol substitution products with four OH groups (G-PDMS-G-1, 2, and 3) are shown in Table 1. The Mn of the starting PDMS was calculated from the ${ }^{1} \mathrm{H}$ NMR results for the end group. The Mn, Mw, and PDI of the starting and product PDMS molecules were determined using GPC. Considering only the terminal change, the molecular weight was expected to increase by 148.16 after the two glycidols were added via substitution. However, the product demonstrated a greater increase in molecular weight. A side reaction produced by the siloxane anion generated by the terminal substitution process of hydroxyl-terminated PDMS appears to have caused disproportionation, resulting in a widening of the molecular weight distribution $[27,28]$. 
Table 1. Molecular weights of dihydroxy-terminated PDMS (OH-PDMS-OH-1, 2, and 3) and glycidolterminated PDMS (G-PDMS-G-1, 2, and 3) by ${ }^{1} \mathrm{H}$ NMR and GPC.

\begin{tabular}{ccccc}
\hline Entry & Mn (NMR) & Mn (GPC) & Mw (GPC) & PDI (GPC) \\
\hline HO-PDMS-OH-1 & 438 & 686 & 804 & 1.17 \\
HO-PDMS-OH-2 & 3295 & 3021 & 4368 & 1.51 \\
HO-PDMS-OH-3 & 3450 & 3043 & 4902 & 1.61 \\
\hline G-PDMS-G-1 & & 883 & 969 & 1.10 \\
G-PDMS-G-2 & & 6499 & 9671 & 1.49 \\
G-PDMS-G-3 & & 6712 & 8831 & 1.32 \\
\hline
\end{tabular}

The water contact angle was measured to confirm the hydrophilicity generated by substituting glycidol at the end of linear HO-PDMS-OH. The HO-PDMS-OH and G-PDMS$\mathrm{G}$ samples were spin-coated on slide glass at $5000 \mathrm{rpm}$ for $40 \mathrm{~s}$ without curing, which could have caused molecular changes. Figure 4A,B are photographs of a water droplet on the surfaces of HO-PDMS-OH-1 and G-PDMS-G-1, which show that the contact angle has changed significantly. Figure $4 \mathrm{C}$ is a graph showing the droplet contact angle of each siloxane polyol. These data indicate that the contact angle increases as the molecular weight increases in the case of HO-PDMS-OH $(48.1 \pm 0.45,58.9 \pm 6.8$, and $64 \pm 0.3)$. The contact angles of G-PDMS-G-1, 2, and 3 were $36.6 \pm 0.9,41.4 \pm 1.3$, and $42.7 \pm 4.25$. These angles are smaller than those of HO-PDMS-OH-1, 2, and 3 by 11.5, 17.5, and 21.3, respectively. Therefore, the hydrophilicity improved with increasing $\mathrm{OH}$ concentration.

(A)

(B)
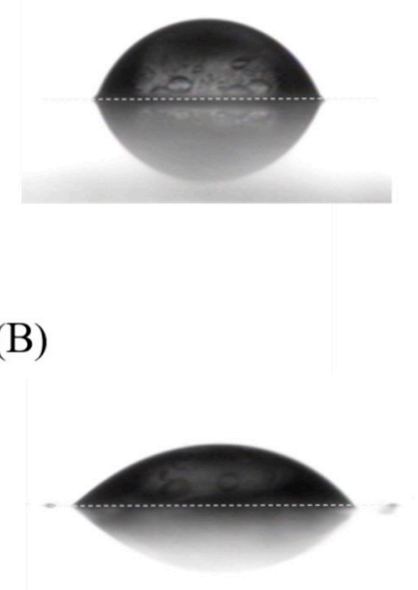

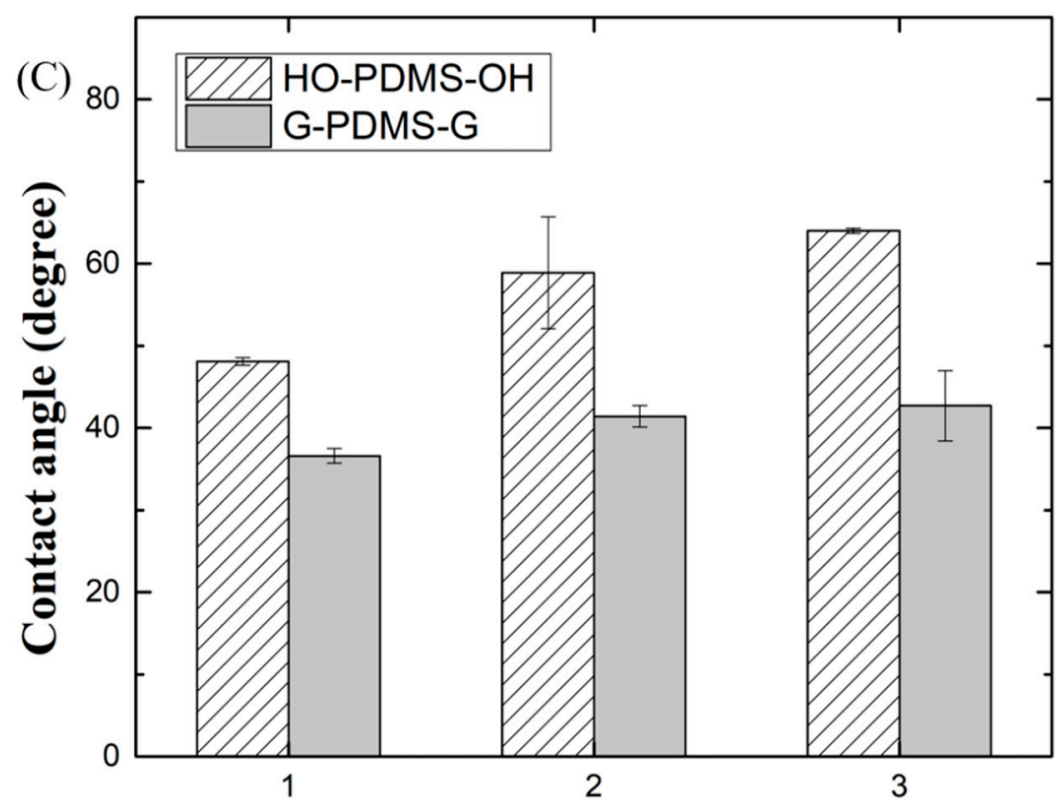

Figure 4. Photo of water droplets on (A) HO-PDMS-OH-1 coated surface and on (B) G-PDMS-G-1 coated surface. (C) Graph showing contact angle of water droplet on HO-PDMS-OH and G-PDMS-G.

The viscosity changes of HO-PDMS-OH and G-PDMS-G upon glycidol substitution are presented in Table 2. The contact angle data for hydroxy-terminated PDMS indicate that longer HO-PDMS-OH siloxane chains correlate with higher hydrophobicities and viscosities. All G-PDMS-G viscosities decreased by $22.77,34.0$, and $88.36 \mathrm{cP}$ compared to those of HO-PDMS-OH, and the sample with the highest molecular weight exhibited a high viscosity difference. Therefore, glycidol substitution is assumed to increase the hydrophilicity. Although the diol substituted hydroxy terminal structure and the increased hydrophilicity were confirmed, the expected molecular weight reproduction and accurate end group analysis were insufficient, and further research is needed. 
Table 2. Viscosities of dihydroxy PDMS and glycidol-terminated PDMS.

\begin{tabular}{ccccc}
\hline & \multicolumn{2}{c}{ HO-PDMS-OH } & \multicolumn{2}{c}{ G-PDMS-G } \\
\cline { 2 - 5 } & Viscosity $(\mathbf{c P})$ & Temperature $\left({ }^{\circ} \mathbf{C}\right)$ & Viscosity (cP) & Temperature $\left({ }^{\circ} \mathbf{C}\right)$ \\
\hline 1 & 34.74 & 21.7 & 11.97 & 21.8 \\
2 & 98.10 & 21.4 & 53.1 & 21.4 \\
3 & 109.2 & 21.4 & 20.84 & 21.2 \\
\hline
\end{tabular}

\section{Conclusions}

In this study, G-PDMS-G-1, G-PDMS-G-2, and G-PDMS-G-3 were synthesized with four $\mathrm{OH}$ groups by diol substitution at both ends of hydroxy-terminated PDMSs (HOPDMS-OH-1, HO-PDMS-OH-2, and HO-PDMS-OH-3). The ${ }^{1} \mathrm{H}$ NMR results confirmed that glycidol was substituted at both ends. In addition, the water contact angles of GPDMS-G on both sides decreased by $24 \%, 30 \%$, and $33 \%$, respectively, compared to those of dihydroxyl PDMS. In addition, the viscosities of G-PDMS-G also decreased compared to those of HO-PDMS-OH in all cases, by $24 \%, 30 \%$, and $33 \%$. Based on these results, it was concluded that increased $\mathrm{OH}$ concentration improves hydrophilicity. Therefore, G-PDMS-G with high hydrophilicity is expected to be useful in the field of PU synthesis, in which flame retardancy is achieved by reacting with diisocyanate.

Supplementary Materials: The following are available online at https:/ / www.mdpi.com/article / 10.3390/ma15030753/s1, Figure S1: 14C NMR spectra of (A) G-PDMS-G-3, (B) G-PDMS-G-2, and (C) glycidol; Figure S2: FTIR spectra of HO-PDMS-OH-3 and G-PDMS-G-3.

Author Contributions: Conceptualization, K.E.P. and S.Y.; Data curation, L.-H.Y.; Formal analysis, L.-H.Y. and K.E.P.; Methodology, K.E.P.; Supervision, S.Y.; Visualization, L.-H.Y.; Writing-original draft, L.-H.Y. and K.E.P.; Writing-review \& editing, L.-H.Y. and S.Y. All authors have read and agreed to the published version of the manuscript.

Funding: This research received no external funding.

Institutional Review Board Statement: Not applicable.

Informed Consent Statement: Not applicable.

Data Availability Statement: The data presented in this study are available upon request from the corresponding author.

Acknowledgments: This work was supported by the Technology Innovation Program (20010315) funded by the Ministry of Trade, Industry \& Energy (MOTIE, Republic of Korea) and also by the C1 Gas Refinery Program (2018M3D3A1A01018006) through the National Research Foundation of Korea (NRF) funded by the Ministry of Science, ICT, and Future Planning, Republic of Korea.

Conflicts of Interest: The authors declare no conflict of interest.

\section{References}

1. Dow Corning Corp. An Overview of Polydimethylsiloxane (PDMS) Fluids in the Environment; Dow Corning Corp.: Midland, MI, USA, 1997.

2. Chaumont, P.; Beinert, G.; Herz, J.; Rempp, P. Synthesis and characterization of multiblock copolymers containing poly (dimethyl siloxane) blocks. Polymer 1981, 22, 663-666. [CrossRef]

3. Zaman, Q.; Zia, K.M.; Zuber, M.; Mabkhot, Y.N.; Almalki, F.; Hadda, T.B. A conprehensive review on synthesis, chracterization, and applications of polydimethylsiloxane and copolymers. Int. J. Plast. Technol. 2019, 23, 261-282. [CrossRef]

4. Singh, H.; Jain, A. Ignition, combustion, toxicity, and fire retardancy of polyurethane foams: A comprehensive review. J. Appl. Polym. Sci. 2009, 111, 1115-1143. [CrossRef]

5. Cui, M.; Li, J.; Chen, W.; Hong, W.; Chen, Y.; Xiang, J.; Yan, J.; Fan, H. A halogen-free, flame retardant, waterborne polyurethane coating based on the synergistic effect of phosphorus and silicon Prog. Org. Coat. 2021, 158, 106359. [CrossRef]

6. Song, L.; Yang, H.; Li, D.; Jiang, Q.; Zeng, D. Polydimethylsiloxane/monomer casting nylon copolymers: Preparation, flameretardant properties, and wear-resistant properties J. Appl Polym. Sci. 2020, 137, 48753. [CrossRef]

7. Zhang, Z.; Song, X.; Cui, L.; Qi, Y. Synthesis of polydimethylsiloxane-modified polyurethane and the structure and properties of its antifouling coatings. Coatings 2018, 8, 157. [CrossRef] 
8. Cui, Y.; Li, Y.; Huan, D.; Zeng, D.; Yang, Y.; Zhu, C.; Wang, J. Fabrication of silicone modified polyurethane matrix superhydrophobic coating with hydroxy-terminated polydimethylsiloxane modified $\mathrm{SiO}_{2}$ nanoparticles. Polym. Plast. Technol. Mater. 2022.

9. Arkles, B. Hydrophobicity, Hydrophilicity and Silane Surface Modification; Gelest Inc.: Morrisville, PA, USA, 2011.

10. Janik, H.; Sienkiewicz, M.; Kucinska-Lipka, J. Handbook of Thermoset Plastics: 9. Polyurethanes; Elsevier: Amsterdam, The Netherlands, 2013.

11. Abbasi, F.; Mirzadeh, H.; Katbab, A.A. Modification of polysiloxane polymers for biomedical applications: A review. Polym. Int 2001, 50, 1279-1287. [CrossRef]

12. Boehm, P.; Frey, H.; Menke, T.J. Hydrophilic Polyorganosiloxanes. United States Patent Application Publication US $2013 / 0123530$ A1, 16 May 2013.

13. Miyanaga, S. Silicone Modified with Branched Polyglycerol. European Patent Office EP 1489 128 A1, 22 December 2004.

14. Miyanaga, S. Silicone Modifiee par Polyglycerol Ramifie. European Patent Office EP 1489128 B1, 21 September 2011.

15. Tamura, S.; Sawayama, S.; Hori, S. Manufacturing Method for High-Purity Glycerol Derivative-Modified Silicone. United States Patent Application Publication, US 2016/0052944 A1, 25 February 2016.

16. Wagner, R.; Richter, L.; Wu, Y.; Weifimüller, J.; Reiners, J.; Klein, K.D.; Schaefer, D.; Stadtmüller, S. Carbohydrate-Modified Siloxane Surfactants: The Effect of Substructures on the Wetting Behavior on Non Polar Solid Surfaces. Organosilicon Chem. III Mol. Mater. 2005, 510-514. [CrossRef]

17. Wagner, R.; Richter, L.; Wersig, R.; Schmaucks, G.; Weiland, B.; Weissmüller, J.; Reiners, J. Silicon-modified carbohydrate surfactants I: Synthesis of siloxanyl moieties containing straight-chained glycosides and amides. Appl. Organomet. Chem. 1996, 10, 421-435. [CrossRef]

18. Jonas, G.; Stadler, R. Polysiloxanes with statistically distributed glucose and galactose units, 1 . Synthesis and thermal characterization. Die Makromol. Chem. Rapid Commun. 1991, 12, 625-632. [CrossRef]

19. Jonas, G.; Stadler, R. Carbohydrate modified polysiloxanes II. Synthesis via hydrosilation of mono-, di-and oligosaccharide allylglycosides. Acta Polym. 1994, 45, 14-20. [CrossRef]

20. Zhan, X.L.; Luo, Z.H.; Zhang, Q.H.; Chen, B.; Chen, F.Q. Synthesis and characterization of $\alpha-\{3-[(2,3-$ dihydroxy) propoxy] propyl\}- $\omega$-butyl-polydimethylsiloxanes. Chin. Chem. Lett. 2009, 20, 729-732. [CrossRef]

21. Jang, S.-H.; Baek, S.-S.; Hwang, S.-H. Preparation, Surface Characteristics and Physical Properties of Polyurethane-based Gel-coat Containing Reactive Silicone Oligomers. Elastomers Compos. 2016, 51, 269-274. [CrossRef]

22. Clayden, J.; Warren, S. Solutions Manual to Accompany Organic Chemistry; Oxford University Press: Oxford, UK, 2013.

23. Weiss, M.E.R. A Kinetic Model for the Anionic Ring-Opening Polymerization of Glycidol. Ph.D. Thesis, Freie Universität, Berlin, Germany, 2012.

24. Cucciniello, R.; Ricciardi, M.; Vitiello, R.; Di Serio, M.; Proto, A.; Capacchione, C. Synthesis of monoalkyl glyceryl ethers by ring opening of glycidol with alcohols in the presence of lewis acids. ChemSusChem 2016, 9, 3272-3275. [CrossRef] [PubMed]

25. Pavia, D.L.; Lampman, G.M.; Kriz, G.S.; Vyvyan, J.A. Introduction to Spectroscopy; Cengage Learning: Boston, MA, USA, 2008.

26. Helms, E.; Arpaia, N.; Widener, M. Assigning the NMR spectrum of glycidol: An advanced organic chemistry exercise. J. Chem. Educ. 2007, 84, 1328. [CrossRef]

27. Chojnowski, J.; Kaźmierski, K.; Rubinsztajn, S.; Stańczyk, W. Transformation of oligodimethylsiloxanols in the presence of a strong base. Reactivity enhancement of the siloxane bond by the adjacent hydroxyl group. Die Makromol. Chem. Macromol. Chem. Phys. 1986, 187, 2039-2052. [CrossRef]

28. Jones, R.G.; Ando, W.; Chojnowski, J. Silicon-Containing Polymers: The Science and Technology of Their Synthesis and Applications; Springer Science \& Business Media: Berlin, Germany, 2013. 